\title{
Diffractive Dijet Production with Large Rapidity Gap in Deep-Inelastic Scattering at HERA
}

\author{
Boris Pokorný ${ }^{\dagger}$ \\ Charles University in Prague - Institute of Particle and Nuclear Physics \\ V Holešovičkách 2, 18000 Prague 8 - Czech Republic \\ E-mail: pokorny@mail.desy.de
}

\begin{abstract}
A measurement of differential dijet cross sections in diffractive deep inelastic ep scattering based on a data sample of an integrated luminosity of $290 \mathrm{pb}^{-1}$ is presented. The investigated phase space is spanned by the photon virtuality in the range of $4<Q^{2}<100 \mathrm{GeV}^{2}$ and by the fractional proton longitudinal momentum loss $x_{\mathbb{P}}<0.03$. The resulting cross sections are confronted with next-to-leading order QCD predictions based on diffractive parton distribution functions determined in inclusive diffractive measurements.
\end{abstract}

XXII. International Workshop on Deep-Inelastic Scattering and Related Subjects,

28 April - 2 May 2014

Warsaw, Poland

\footnotetext{
* Speaker.

${ }^{\dagger}$ On behalf of H1 Collaboration, supported by SVV 267309 of Charles University in Prague
} 


\section{Introduction}

Diffractive reactions of type $e p \rightarrow e X Y$, where $X$ is a high-mass hadronic final state and $Y$ is either the elastically scattered proton or its low-mass excitation, represent about $10 \%$ of neutral current DIS events observed at HERA. The QCD factorisation theorem [1,2] states the calculation of the diffractive DIS cross section factorises into hard scattering matrix elements calculable within the perturbative approach and into non-perturbative diffractive parton distribution functions (DPDF) introduced in analogy with inclusive DIS. DPDFs are obtained in QCD fits of inclusive diffractive data [3] and are successfully used to predict cross sections for diffractive production of jets, charm and vector mesons.

DPDFs are dominated by the gluon component which is determined indirectly via scaling violations in the inclusive diffractive measurements. Exclusive measurements of production of jets or heavy quarks are sensitive directly to the gluon component of DPDFs as the underlying process is mostly the boson-gluon fusion (BGF) [4].

Final states of diffractive collisions are characterised by the clear separation of the systems $X$ and $Y$ in rapidity. Diffractive events may be identified either by the large rapidity gap (LRG) method, where no energy deposit above the noise level is required in the forward region of the detector, or by direct detection of the scattered proton. The production of dijets in diffractive DIS has been studied at HERA using both the LRG method [5,6] and the direct scattered proton tagging [7]. The results of the previous dijet measurements are well reproduced by next-to-leading order (NLO) QCD calculations based on DPDF extracted from the inclusive diffractive data.

A new measurement of the dijet production in diffractive DIS is provided by H1. The luminosity of the analysed data is unprecedentedly high, six times higher than [5], and, in order to maximise the aim of the high statistics, the data are corrected to the level of stable hadrons using the regularised unfolding. The measured cross sections are compared to the NLO QCD predictions evaluated with input DPDFs determined in previous inclusive diffractive measurements at H1 [3].

\section{Experimental Procedure}

The data of the total luminosity of $290 \mathrm{pb}^{-1}$, collected by the $\mathrm{H} 1$ detector $[8,9]$, are analysed in the presented measurement. The visible phase space for the diffractive dijet production is defined by the following kinematic boundaries:

$$
\begin{gathered}
4<Q^{2}<100 \mathrm{GeV}^{2}, 0.1<y<0.7 \\
|t|<1 \mathrm{GeV}^{2}, M_{Y}<1.6 \mathrm{GeV} \\
x_{\mathbb{P}}<0.03 \\
p_{\mathrm{T}, 1}^{*}>5.5 \mathrm{GeV}, p_{\mathrm{T}, 2}^{*}>4.0 \mathrm{GeV} \\
-1<\eta_{1,2}^{\mathrm{LAB}}<2
\end{gathered}
$$

The DIS events are selected by identification of the scattered electron. The measurement is constrained by (2.1) in the photon virtuality $Q^{2}$ and in the inelasticity $y$ in order to achieve the precise determination of the scattered electron four-momentum. 
A sub-sample of diffractive events is selected using the LRG method where signals left in the forward components of the detector are required to be below noise levels. The system $X$ is thus isolated in the main part of the detector while the system $Y$ escapes undetected. The momentum transfer $t$ and the mass $M_{Y}$ are not reconstructed and the resulting cross sections are integrated in the limits (2.2) which are imposed by efficiencies of the sub-detectors used in the LRG selection. The relative proton momentum loss $x_{\mathbb{P}}$ is constrained by (2.3) to ensure the clear separation of the systems $X$ and $Y$ is feasible.

Jets are reconstructed by the $k_{T}$-algorithm with $R=1[10]$ in the $\gamma^{*}-p$ frame where the virtual photon collides head-on with the proton. The leading and sub-leading jets are constrained in transverse momenta $p_{\mathrm{T}, 1}^{*}$ and $p_{\mathrm{T}, 2}^{*}$ in the $\gamma^{*}-p$ frame (2.4) and in pseudorapidities $\eta_{1}^{\mathrm{LAB}}$ and $\eta_{1}^{\mathrm{LAB}}$ in the laboratory frame (2.5).

The data are corrected to the hadron-level using the matrix unfolding with regularisation [11, 12,13] implemented in the program TUnfold [14]. The statistical correlations between the different measurement bins as well as migrations around the phase space boundaries are treated within the unfolding procedure. The migration matrix is determined in the MC simulation using the RAPGAP event generator [15].

\section{Theoretical Predictions}

Cross sections for the diffractive DIS dijet production are calculated using the program NLOJET++ [16] adjusted for the diffractive regime [17]. The calculation is based on the factorisation theorem. The hard scattering matrix elements are calculated up to NLO in the powers of the strong coupling $\alpha_{s}\left(\mu_{r}\right)$ within the $\bar{M} S$-scheme using five active flavors and the two-loop approximation for the running of $\alpha_{s}$. The world average value $\alpha_{s}\left(m_{Z}\right)=0.118$ corresponding to $\Lambda_{5}=0.228 \mathrm{GeV}$ is considered. The matrix elements are convoluted with H1 2006 Fit B [3] DPDF. The renormalisation and factorisation scales are defined as $\mu_{r}=\mu_{f}=\sqrt{p_{\mathrm{T}, 1}^{* 2}+Q^{2}}$. The calculation yields parton-level cross sections which are corrected for the hadronisation effects using the MC simulation with the RAPGAP event generator.

\section{Results}

The integrated cross section in the phase space given by constrains (2.1)- (2.5) is measured to be

$$
\sigma^{2 j e t}(e p \rightarrow e X Y)=69 \pm 2 \text { (stat.) } \pm 8 \text { (syst.) pb . }
$$

The total cross section is compared to the NLO QCD prediction

$$
\left.\sigma_{N L O}^{2 j e t}(e p \rightarrow e X Y)=66_{-17}^{+19}(\text { scale })_{-16}^{+9}(\mathrm{DPDF}) \pm 3 \text { (hadr. }\right) \mathrm{pb} .
$$

The prediction is compatible with the measurement within the experimental uncertainties. The uncertainty on the NLO prediction is found to be larger than the total experimental uncertainty.

Figure 1 shows the differential cross sections measured as a function of $p_{\mathrm{T}, 1}^{*}, Q^{2}, \log x_{\mathbb{P}}$ and $z_{\mathbb{P}}$ compared to the NLO QCD predictions corrected for the hadronisation effects. The shapes of the displayed distributions are well described within the uncertainties. The high statistical uncertainty 
in the highest bin of $p_{\mathrm{T}, 1}^{*}$ arises due to the statistical correlations between the measured bins. The QCD prediction in the highest bin of $z_{\mathbb{P}}$ is biased with the large uncertainty of the DPDF and only the upper bound for the cross section is thus predicted. The cross sections measured differentially as a function of $\left\langle p_{\mathrm{T}}^{*}\right\rangle, p_{\mathrm{T}, 2}^{*}, y$ and $\Delta \eta^{*}=\left|\eta_{1}^{*}-\eta_{2}^{*}\right|$ are displayed in Figure 2. The differential cross sections are determined with experimental uncertainties which are lower than the uncertainties of the NLO QCD calculation.

\section{Conclusion}

The cross sections for the dijet production in diffractive DIS are measured with the LRG selection and are found to be well described by the NLO QCD predictions based on DPDF H1 2006 Fit B. Due to the high luminosity of the analysed data, the achieved experimental precision is higher than the precision of the theory predictions.

\section{References}

[1] L. Trentadue and G. Veneziano. Phys. Lett. B, 323:201, (1994).

[2] J.C. Collins. Phys. Rev. D, 57:3051, (1998).

[3] A. Aktas et al. [H1 Collaboration]. Eur. Phys. J. C, 48:715-748, (2006), [arXiv:hep-ex/0606004].

[4] S. Chekanov et al. [ZEUS Collaboration]. Eur. Phys. J. C, 42:1.

[5] C. Adloff et al. [H1 Collaboration]. Eur. Phys. J. C, 20, (2001) [hep-ex/0012051].

[6] S. Chekanov et al. [ZEUS Collaboration]. Eur. Phys. J. C, 52:813-832, 2007.

[7] F. D. Aaron et al. [H1 Collaboration]. Phys. J. C, 72:1970, (2011) [arXiv:1111.0584].

[8] I. Abt et al. [H1 Collaboration]. Nucl. Instrum. Meth. A, 386:310.

[9] R. Appuhn et al. [H1 SPACAL Group]. Nucl. Instrum. Meth. A, 386:397.

[10] Y. Dokshitzer S. Catani and B. Weber. Phys. Lett. B, 285:291.

[11] D.L.Phillips. Assoc.Comput.Mach, 9:84.

[12] A.N.Tikhonov. Soviet Math. Dokl., 4:1035, (1963), English translation in Dokl.Akad.Nauk SSSR, 151 (1963) 501.

[13] V.Blobel. Proceedings of the Conference on Advanced Statistical Techniques in Particle Physics, Durham, England, page 258, (2002) [hep-ex/0208022].

[14] S.Schmitt. JINST, 7:T10003, (2012).

[15] H. Jung. Comput. Phys. Commun., 86:147.

[16] Z. Nagy and Z. Trocsanyi. Phys. Rev. Lett., 87, (2001) [hep-ph/0104315].

[17] G. Ingelman and P. Schlein. Phys. Lett. B, 152:256, (1985). 
† H1 Data

NLO H1 2006 Fit B $\otimes\left(1+\delta_{h a d}\right)$

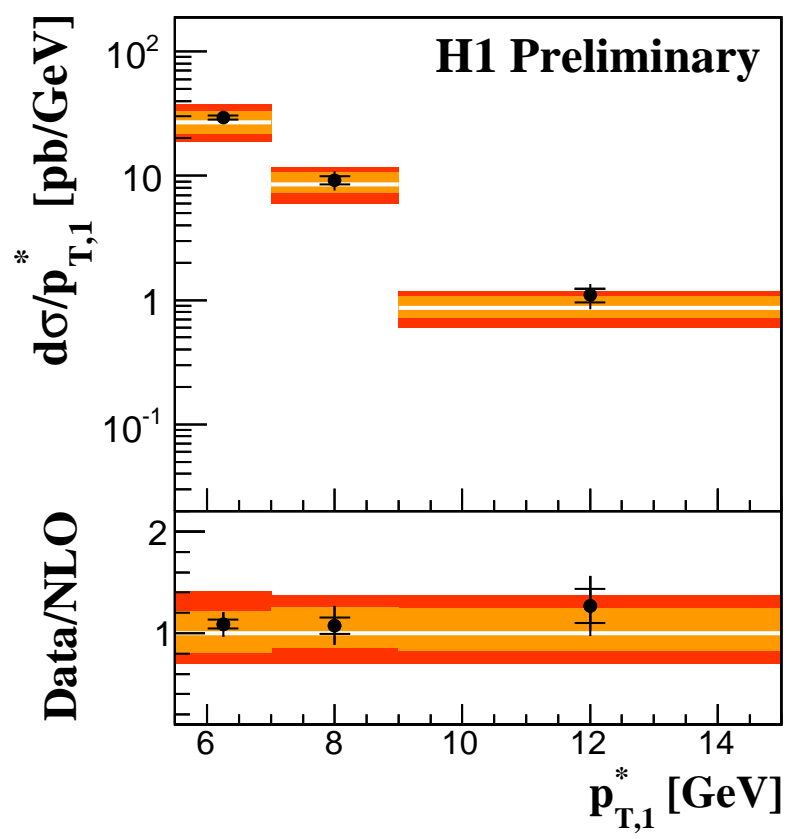

† H1 Data

NLO H1 2006 Fit B $\otimes\left(1+\delta_{h a d}\right)$

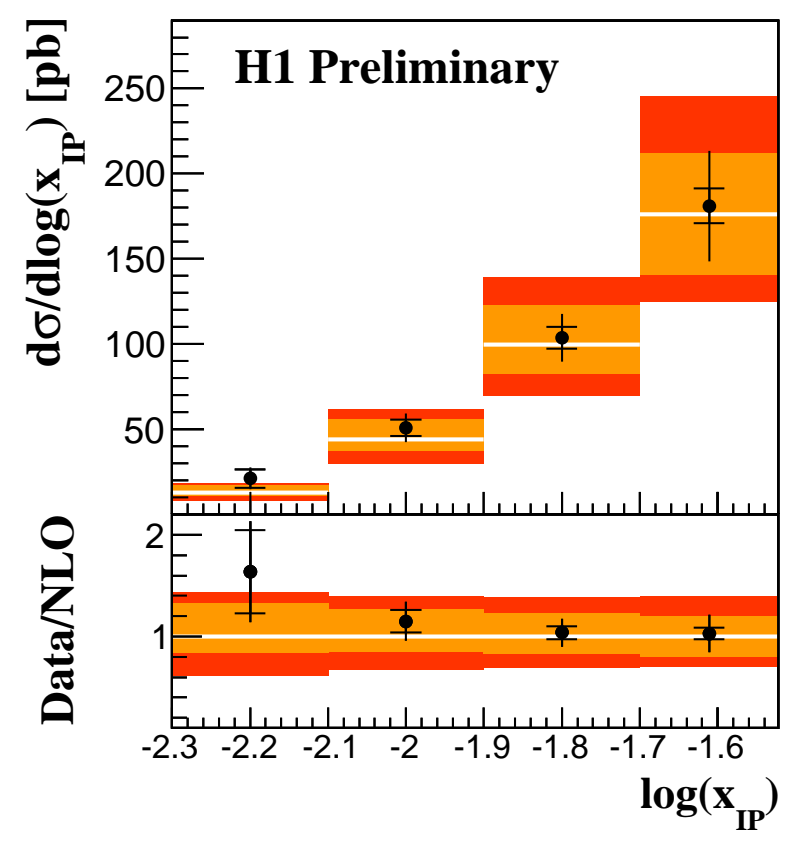

H1 Data

NLO H1 2006 Fit B $\otimes\left(1+\delta_{\text {had }}\right)$

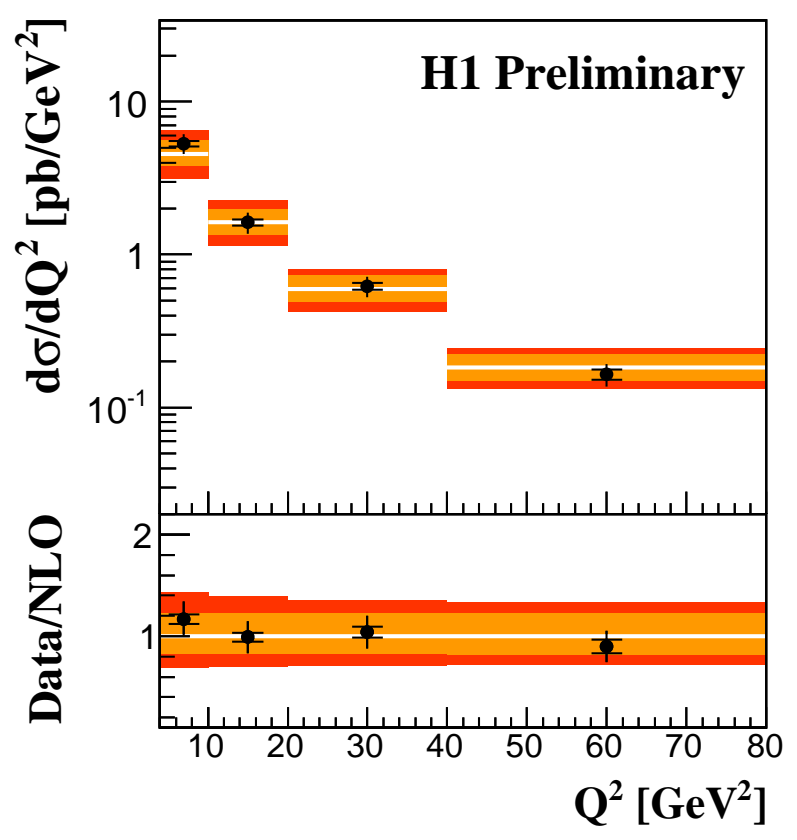

† H1 Data

NLO H1 2006 Fit B $\otimes\left(1+\delta_{\text {had }}\right)$

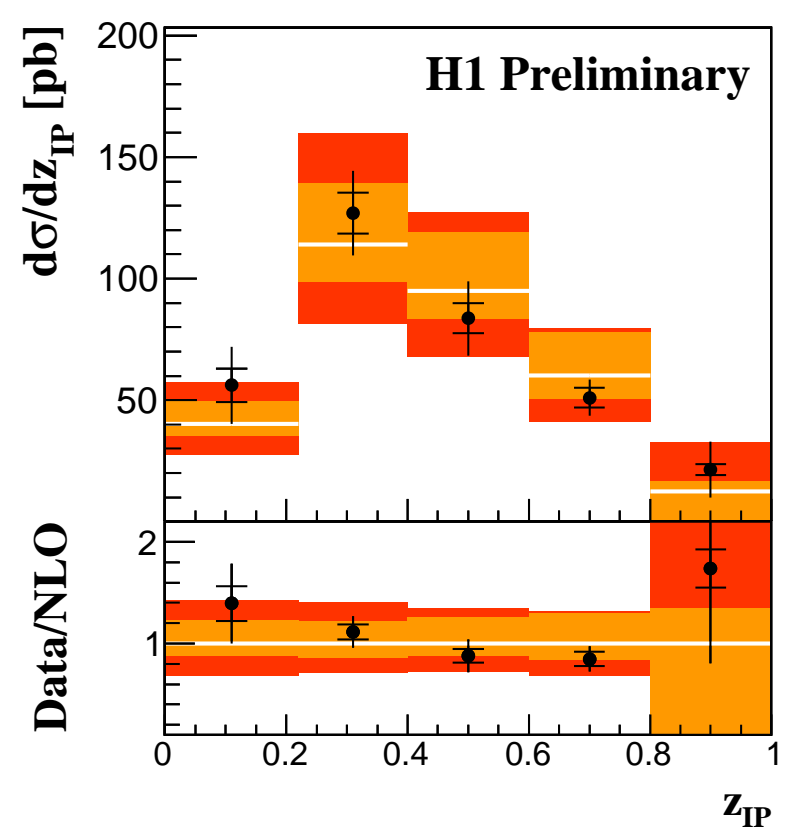

Figure 1: Diffractive dijet differential cross section as a function of $p_{T, 1}^{*}, Q^{2}, \log x_{\mathbb{P}}$ and $z_{\mathbb{P}}$. The inner error bars of the data points represent the statistical uncertainty while the outer error bars include the systematic uncertainties added in quadrature. The NLO QCD prediction based on the DPDF set H1 2006 B is displayed as the white line with the inner band (orange) indicating the uncertainty of the hadronisation and DPDF fit added in quadrature. The outer band (red) includes the QCD scale uncertainty. 
† H1 Data

NLO H1 2006 Fit B $\otimes\left(1+\delta_{\text {had }}\right)$
H1 Data

NLO H1 2006 Fit B $\otimes\left(1+\delta_{\text {had }}\right)$

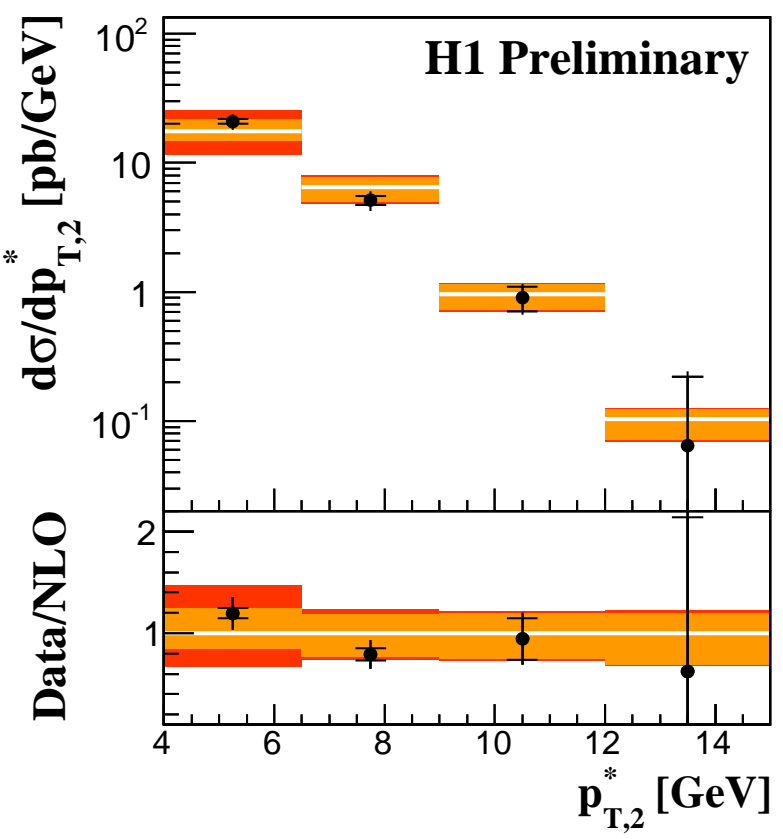

† H1 Data

NLO H1 2006 Fit B $\otimes\left(1+\delta_{\text {had }}\right)$

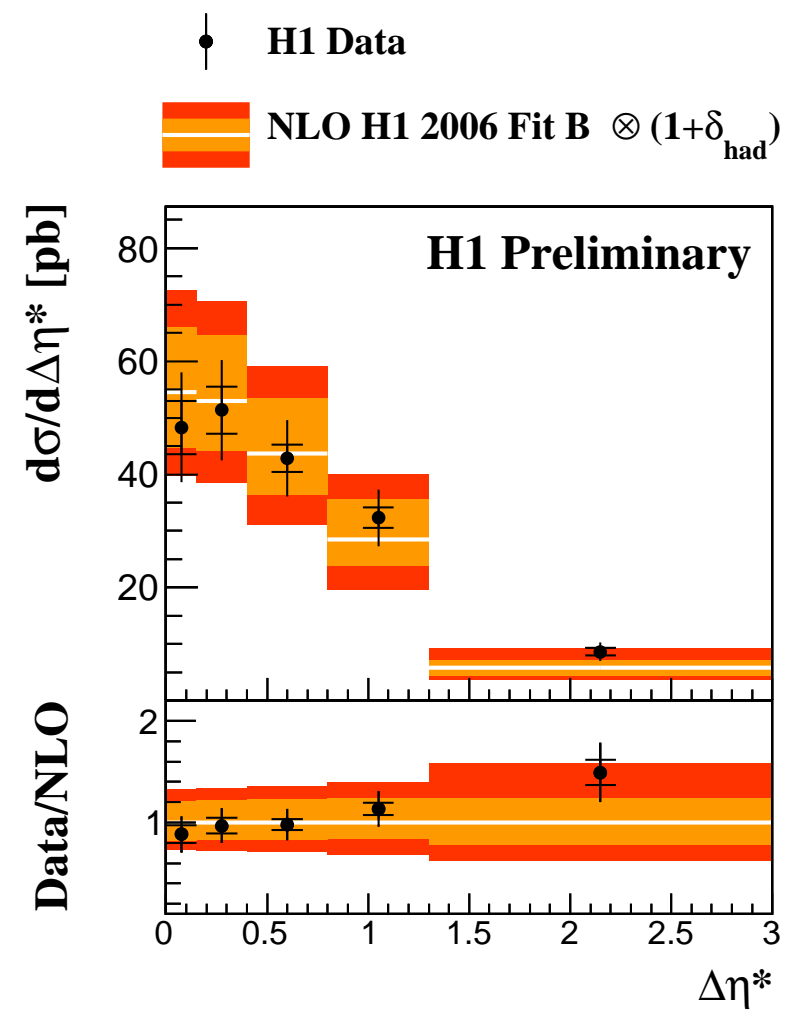

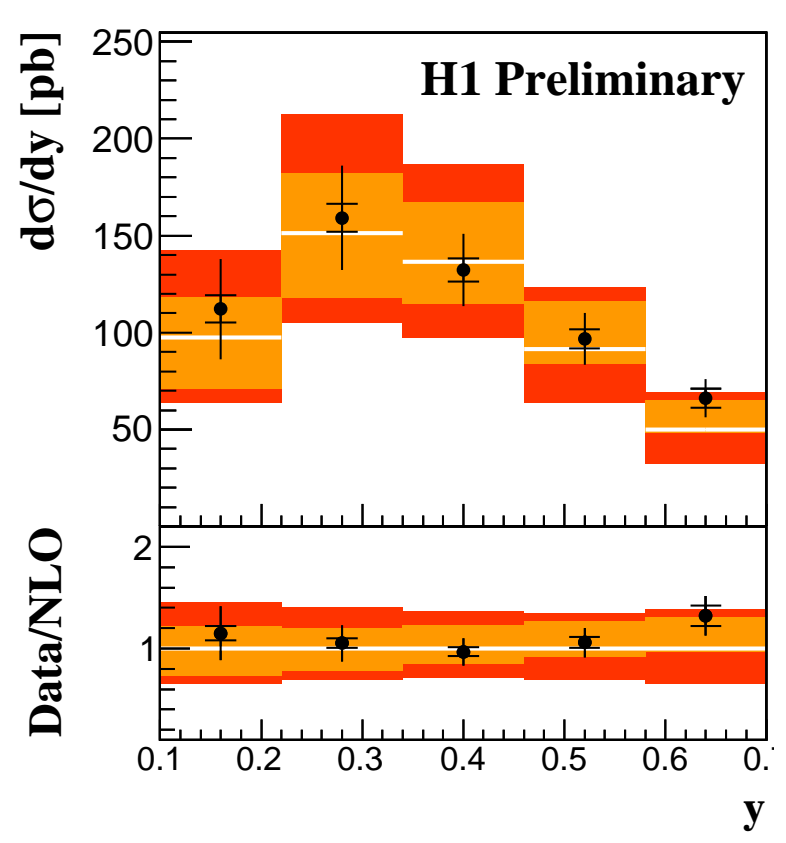

Figure 2: Diffractive dijet differential cross section as a function of $\left\langle p_{\mathrm{T}}^{*}\right\rangle, p_{\mathrm{T}, 2}^{*}, y$ and $\Delta \eta^{*}$. The inner error bars of the data points represent the statistical uncertainty while the outer error bars include the systematic uncertainties added in quadrature. The NLO QCD prediction based on the DPDF set H1 2006 B is displayed as the white line with the inner band (orange) indicating the uncertainty of the hadronisation and DPDF fit added in quadrature. The outer band (red) includes the QCD scale uncertainty. 